\title{
Neoadjuvant chemotherapy with gemcitabine-based regimens improves the prognosis of node positive resectable pancreatic head cancer
}

\author{
HIDEHIRO TAJIMA $^{1}$, TETSUO OHTA ${ }^{1}$, MITSUYOSHI OKAZAKI ${ }^{1}$, TAKAHISA YAMAGUCHI ${ }^{1}$, \\ YOSHINAO OHBATAKE ${ }^{1}$, KOICHI OKAMOTO ${ }^{1}$, SHINICHI NAKANUMA ${ }^{1}$, JUN KINOSHITA ${ }^{1}$, \\ ISAMU MAKINO $^{1}$, KEISHI NAKAMURA ${ }^{1}$, TOMOHARU MIYASHITA ${ }^{1}$, HIROYUKI TAKAMURA ${ }^{1}$, \\ ITASU NINOMIYA ${ }^{1}$, SACHIO FUSHIDA $^{1}$ and HIROYUKI NAKAMURA ${ }^{2}$ \\ Departments of ${ }^{1}$ Gastroenterological Surgery, and ${ }^{2}$ Environmental and Preventive Medicine, \\ Graduate School of Medical Science, Kanazawa University, Kanazawa, Ishikawa 920-8641, Japan
}

Received December 17, 2018; Accepted May 16, 2019

DOI: $10.3892 / \mathrm{mco} .2019 .1867$

\begin{abstract}
The effectiveness of preoperative (neoadjuvant) chemotherapy (NAC) for resectable pancreatic ductal adenocarcinoma (PDAC) remains unclear. The present study retrospectively evaluated the efficacy of NAC with gemcitabine (GEM)-based regimens or GEM monotherapy for resectable PDAC. Between 2006 and 2015, NAC with GEM was performed in 52 cases (head 31, and body and tail 21) and compared with 34 resection-only cases serving as controls (head 20, and body and tail 14). According to the Response Evaluation Criteria In Solid Tumors guidelines, the treatment effect was a partial response in 5 cases, stable disease in 45 cases, and progressive disease in 2 cases. Maximum standardized uptake values and carbohydrate antigen (CA19-9) values were significantly reduced after preoperative chemotherapy. Using the Evans grading system, the treatment effect was grade I in 31 patients, grade IIa in 8 , and grade IIb in 3 cases. There were significant differences in the overall survival rate between the NAC and control groups, only in the patients with node-positive pancreatic head cancer. Significantly higher CA19-9 values in peripheral blood and higher lymph node metastasis and plexus invasion rates were observed in early-recurring cases within a year. The preoperative CA 19-9 cutoff value as an early recurrence risk factor was calculated as $30 \mathrm{U} / \mathrm{ml}$ in the NAC group and $88 \mathrm{U} / \mathrm{ml}$ in the control group. NAC with GEM prolonged survival in patients with node-positive pancreatic head cancer. High CA19-9 values before operation, lymph node metastases
\end{abstract}

Correspondence to: Dr Hidehiro Tajima, Department of Gastroenterological Surgery, Graduate School of Medical Science, Kanazawa University, 13-1 Takaramachi, Kanazawa, Ishikawa 920-8641, Japan

E-mail: hidetaji@staff.kanazawa-u.ac.jp

Key words: pancreatic cancer, resectable, gemcitabine, neoadjuvant chemotherapy and plexus invasion were risk factors for early tumor recurrence after surgery. Preoperative chemotherapy would be necessary for resectable pancreatic head cancer as lymph node metastasis was observed in $>60 \%$ with resectable PDAC. Moreover, if normalization of CA19-9 values is not achieved with NAC, extension of preoperative chemotherapy should be considered as for borderline resectable PDAC cases.

\section{Introduction}

Pancreatic ductal adenocarcinoma (PDAC) is a fatal disease with an overall survival (OS) rate of $<6 \%(1,2)$. Although the advent of $n a b$-paclitaxel (nab-PTX) plus gemcitabine (GEM) therapy ( $\mathrm{GnP}$ therapy) and 5-FU, leucovorin, irinotecan and oxaliplatin (FOLFIRINOX) therapy improved treatment outcome of unresectable PDAC $(3,4)$, surgery is the only method to achieve long-term survival. Curative (R0) resection comprising wide lymph node dissection and complete removal of the extrapancreatic nerve plexus of the superior mesenteric artery (SMA) or celiac axis (5-10) has been shown to be one of the key factors influencing survival of patients with PDAC. However, even in patients who undergo resection, 5 -year survival is poor at $<30 \%$ and the prognosis of PDAC has not improved. Nimura et al (11), reported that extended lymphadenectomy does not improve prognosis in pancreatic head cancer. These disappointing results indicate that surgery alone is inadequate and the poor survival is likely attributable to early hematogenous spread, because in most patients' metastases are present at the time of surgery (12). Investigation of postoperative adjuvant chemotherapy is based on this hypothesis. Oettle et al (13), reported that adjuvant chemotherapy with GEM produced a statistically significant improvement in OS. Recently, the JASPAC-01 study in Japan showed that S-1, an oral fluoropyrimidine analogue, confers significantly improved OS and recurrence-free survival after pancreatic cancer resection compared with GEM (14).

A major drawback of adjuvant therapy for PDAC is that $20-30 \%$ of patients are ineligible to receive the designated therapy because of postoperative complications, such as 
delayed surgical recovery, patient refusal, comorbidity, or early disease recurrence (15-17). This could be overcome by the preoperative (neoadjuvant) chemotherapy (NAC) or chemoradiotherapy so that more patients can receive potentially beneficial treatment. Other theoretical advantages of this approach include the following: Early treatment of micrometastases; sparing those who already have occult metastases the morbidity and mortality associated with major surgery if disseminated disease becomes apparent at the time of reassessment; reduced risk of tumor seeding at the time of surgery; and improved tolerance compared with postoperative therapys.

Potential disadvantages of neoadjuvant therapy include the following: A requirement for biliary decompression before chemotherapy and the potential for complications associated with biliary stents; delayed surgery, allowing progression to an unresectable stage in patients whose disease does not respond to therapy; and the potential for an increase in postoperative complications. Recently, results of randomized clinical trials and data analyses of preoperative therapy for borderline resectable and locally advanced PDAC have been reported (18-22). However, there have been few reports with high evidence levels on preoperative therapy for resectable PDAC.

We have used neoadjuvant chemotherapy (NAC) for resectable PDAC since December 2006, and previously conducted some clinical studies of NAC with a GEM plus S-1 (GS) regimen for resectable PDAC as a pilot study and phase I trial $(23,24)$. From August 2013, NAC with a GnP protocol has been used for resectable PDAC in a pilot clinical trial. GEM monotherapy was performed at the transition of two regimens. We report our local experience and long-term outcomes with NAC with GEM-based regimens for resectable PDAC, compared with those treated with upfront surgery retrospectively. In addition, we evaluate risk factors for recurrence after surgery for potentially resectable PDAC cases in the same period.

\section{Materials and methods}

Patients and NAC regimens. From January 2006 to December 2015, 91 patients with radiologically-proven PDAC considered 'resectable' according to the National Comprehensive Cancer Network (NCCN) guidelines and 86 (50 males and 36 female) patients were operated on at the Department of Gastroenterological Surgery, Kanazawa University Hospital. Five patients did not undergo surgery due to rapid tumor local progression in two cases, distant metastasis detected after preoperative chemotherapy in two cases and a case of portal vein thrombosis caused by biliary drainage during preoperative chemotherapy. In this period, NAC with GEM-based regimens was performed in 52 cases (NAC group) of the 86 resectable PDAC cases, and in the remaining 34 cases, surgery was performed without preoperative chemotherapy (Control group) at the discretion of the attending physician. In 52 cases of NAC group, there were 31 pancreatic head cancer and 21 body and tail cancer. Control group obtained 20 pancreatic head cancer and 14 body and tail cancer. Three types of GEM based regimens, GEM alone, GS, and GnP therapies were adopted in the NAC group. The case numbers of each treatment was 10 cases of GEM alone, 33 cases of GS and 9 cases of GnP. In GEM monotherapy, $1,000 \mathrm{mg} / \mathrm{m}^{2}$ of $\mathrm{GEM}$ was administered as a 30 min intravenous infusion on days 8,15 and 22 of each cycle. The cycle was performed twice every 28 days (Fig. 1A). In GS therapy, S-1 was administered orally postprandially for 14 consecutive days at the dose of $20 \mathrm{mg} / \mathrm{m}^{2} /$ day (from the evening of day 1 to the morning of day 15 ), and $1,000 \mathrm{mg} / \mathrm{m}^{2}$ of GEM was administered as a $30 \mathrm{~min}$ intravenous infusion on days 8 and 15 of each cycle. The cycle was performed twice every 21 days (Fig. 1B). In GnP therapy, 25 or $50 \mathrm{mg} / \mathrm{m}^{2}$ of $n a b$-PTX and $1,000 \mathrm{mg} / \mathrm{m}^{2}$ of GEM were administered as 60 and $30 \mathrm{~min}$ intravenous infusions, respectively, on days 8,15 and 22 of each cycle. The cycle was performed twice every 28 days (Fig. 1C). In all the NAC regimens, surgery was performed $>14$ days after the two cycles of chemotherapy ended.

Written informed consent was obtained from each patient prior to treatment, and the present study was approved by the Ethics Committee of Kanazawa University Hospital (review number: 2799-1). The authors declare that they have no competing interests about this study.

Assessments of efficacy. All the patients with resectable PDAC were diagnosed by multidetector computed tomography (MDCT), magnetic resonance imaging enhanced with gadolinium ethoxybenzyl diethylenetriamine pentaacetic acid (Gd-EOB-DTPA) (EOB-MRI), and 18-fluorodeoxyglucose-positron emission tomography/computed tomography $\left({ }^{18} \mathrm{FDG}-\mathrm{PET} / \mathrm{CT}\right)$ imaging. In the NAC group tumor response was by comparing pretreatment and posttreatment images, and was graded according to the Response Evaluation Criteria in Solid Tumors (RECIST) version 1.0 (25). Complete response (CR) was defined as the disappearance of all clinical evidence of the measurable tumor. Partial response (PR) was defined as a $30 \%$ or greater reduction in the sum of the products of 2 perpendicular diameters of all measurable lesions compared with the baseline values, with no evidence of new lesions. Stable disease (SD) was defined as $<30 \%$ reduction or $<20 \%$ increase in the sum of the products of 2 perpendicular diameters of all measurable lesions compared with the baseline values, with no evidence of new lesions. Progressive disease (PD) was defined as an increase of $20 \%$ or more in the sum of the products of 2 perpendicular diameters of all measurable lesions compared with the baseline values, the appearance of any new lesion, or deterioration in clinical status consistent with disease progression. To assess objective treatment responses, patients were reevaluated with MDCT, EOB-MRI and ${ }^{18} \mathrm{FDG}$-PET/CT after two cycles of preoperative chemotherapy. Carcinoembryonic antigen (CEA), carbohydrate antigen (CA) 19-9, and sialyl-lcat-N-tetraose (DUPAN-2) were measured before and after chemotherapy. In the control group, the same markers were measured before operation.

Pathological diagnosis. All surgically resected specimens were immediately fixed in $10 \%$ neutral-buffered formaldehyde solution. After the specimens had been cut horizontally into 5-mm tissue blocks (26), they were dehydrated and embedded in paraffin. Finally, 5- $\mu \mathrm{m}$ sections were cut and stained with hematoxylin and eosin. Each section was carefully examined using light microscopy. The tumors were evaluated according to the NCCN guidelines of Pancreatic Adenocarcinoma version 3.2017. The grading system of Evans et al (27), was used to 
A

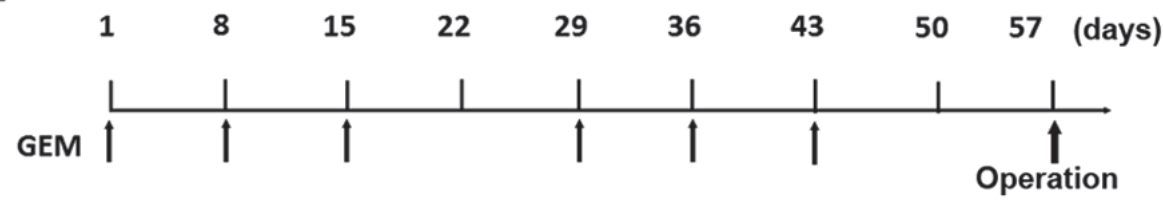

GEM : $1,000 \mathrm{mg} / \mathrm{m}^{2}$

B

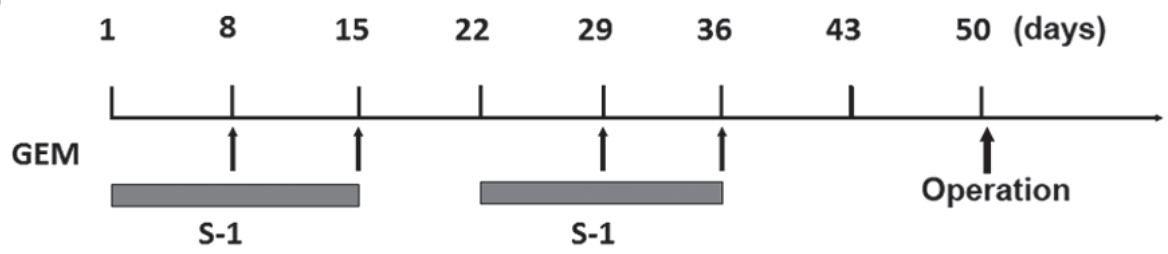

GEM: $800-1,000 \mathrm{mg} / \mathrm{m}^{2}$

$\mathrm{S}-1: 30 \mathrm{mg} / \mathrm{m}^{2} /$ day

\begin{tabular}{|c|c|}
\hline Body surface area & $\mathrm{s}-1$ \\
\hline$<1.34 \mathrm{~m}^{2}$ & $30 \mathrm{mg} /$ day \\
\hline $1.34 \mathrm{~m}^{2} \sim 1.67 \mathrm{~m}^{2}$ & $40 \mathrm{mg} /$ day \\
\hline$>1.5 \mathrm{~m}^{2}$ & $50 \mathrm{mg} /$ day \\
\hline
\end{tabular}

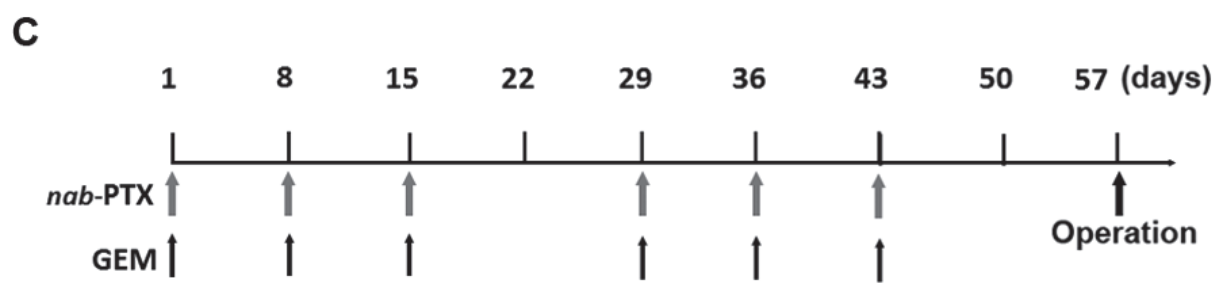

nab-PTX: $25-50 \mathrm{mg} / \mathrm{m}^{2}$

GEM: $1,000 \mathrm{mg} / \mathrm{m}^{2}$

Figure 1. Treatment protocol for NAC with GEM-based regimens. (A) GEM monotherapy. GEM $\left(1,000 \mathrm{mg} / \mathrm{m}^{2}\right)$ was administered on days 1,8 and 15 of the first cycle, and on days 29, 36 and 43 in the second cycle. (B) GEM plus S-1 therapy: S-1 (20-40 mg/m²/day) was given orally for 14 consecutive days, and GEM (1,000 mg/m²) was administered intravenously on days 8 and 15 in the first cycle, and on days 29 and 36 in the second cycle. (C) $n a b$-PTX plus GEM therapy: $25-50 \mathrm{mg} / \mathrm{m}^{2}$ of $n a b$-PTX and $1,000 \mathrm{mg} / \mathrm{m}^{2}$ of GEM were administered intravenously on days 1,8 and 15 in the first cycle, and on days 29,36 and 43 in the second cycle. In all NAC regimens, surgery was performed $>2$ weeks after the 2 treatment cycles. NAC, neoadjuvant chemotherapy; GEM, gemcitabine; $n a b$-PTX, nab-paclitaxel.

assess the pathological effects of preoperative chemotherapy. The degrees of cytological changes and tumor destruction were graded on a scale of I-IV, as follows: Grade I, presence of characteristics cytological changes of malignancy, but little $(<10 \%)$ or no evident tumor cells destruction; grade IIa, destruction of $10-50 \%$ of tumor cells; grade IIb, destruction of $51-90 \%$ of tumor cells; grade III, presence of few $(<10 \%)$ viable tumor cells; grade IIIM, presence of sizeable pools of mucin; grade IV, presence of no viable tumor cells; and grade IVM, presence of acellular pools of mucin.

Patient follow up. After operation, patients were examined for recurrence with enhanced MDCT every 3-4 months and blood tests including tumor marker analysis every month. When recurrence was suspected, ${ }^{18}$ FDG-PET/CT was performed. Two radiologists reviewed the scans. The tumor relapse day was the date when $\mathrm{CT}$ confirmed the recurrence.
Risk factors for early recurrence after surgery. There were 35 recurrences within 1 year after surgery (E group) out of 86 resected PDAC cases from 2006 to 2015. We compared the remaining 51 patients with no relapse or recurrence after $>1$ year (L group), looking for differences in risk factors.

Statistical analyses. Categorical variables were compared using the chi-squared test, Student's $t$-test, and the paired $t$-test. The OS and disease free survival (DFS) rates were calculated from the start of the study treatment until death or the final date of follow up and determined by the Kaplan-Meier method, and the log-rank test was applied for comparison of survival rates between groups. A CA19-9 cutoff value as a risk factor for early recurrence was calculated with receiver operating characteristics (ROC) analysis. All the analyses were performed using commercial software (SPSS ${ }^{\circledR}$ v.23, SPSS Inc., Chicago IL, USA). P<0.05 was considered to indicate a statistically significant difference. 
Table I. Patient characteristics of the NAC and Control groups.

\begin{tabular}{|c|c|c|c|}
\hline Characteristics & NAC group $(n=52)$ & Control group $(n=34)$ & P-value \\
\hline Gender, Male:Female & $29: 23$ & $21: 13$ & 0.582 \\
\hline Median age, years (range) & $65.4(48-82)$ & $68.0(52-84)$ & 0.636 \\
\hline Tumor location, Head:Body and tail & $31: 21$ & 20:14 & 0.942 \\
\hline Tumor size via CT, mm & & & 0.180 \\
\hline Before NAC & $23.3 \pm 6.5$ & $26.6 \pm 9.8$ & \\
\hline After NAC & $22.0 \pm 6.2$ & & \\
\hline SUVmax & & & 0.624 \\
\hline Before NAC & $4.7 \pm 4.2$ & $5.6 \pm 3.7$ & \\
\hline After NAC & $3.0 \pm 3.2^{\mathrm{a}}$ & & \\
\hline CA19-9, U/ml & & & 0.699 \\
\hline Before NAC & $183.5 \pm 329.0$ & $155.9 \pm 223.9$ & \\
\hline After NAC & $93.2 \pm 198.1^{\mathrm{b}}$ & & \\
\hline
\end{tabular}

\section{Results}

Patient characteristics. The characteristics of the NAC group and Control (surgery-only) groups are listed Table I. There were not significant differences in terms of gender, age, tumor location, tumor size on $\mathrm{CT},{ }^{18} \mathrm{FDG}$ maximum standardized uptake value (SUVmax), or in tumor marker (CA19-9) values. Comparisons of resected histopathological findings are shown in Table II. There were no significant differences between the NAC group and the Control group in any histopathological findings (tumor size; serosal, retroperitoneal, neural and plexus invasion rate; lymph node metastasis rate; and R0 rate).

Intraoperative bleeding was $471.9 \pm 344.2 \mathrm{ml}$ in the NAC group and $502.1 \pm 374.6 \mathrm{ml}$ in the Control group. There were no significant differences in the amount of bleeding between the two group $(\mathrm{P}=0.702)$, and the overall postoperative mortality rate was $0 \%$ in both groups. No differences in rates of postoperative complications were found between the two groups. Postoperative adjuvant chemotherapy was performed in 43 of 52 cases $(82.7 \%)$ in the NAC group, and in 13 of 34 cases $(38.2 \%)$ in the Control group $(\mathrm{P}<0.0001)$. There was no difference about side effects among three chemotherapeutic regimens except for epilation of $\mathrm{GnP}$ therapy.

Efficacy of NAC. Five of 52 NAC group patients (9.6\%) showed a PR, 45 (86.5\%) showed SD and 2 (3.9\%) showed PD. In comparison of the clinical objective treatment effects before and after NAC listed in Table I, no significant tumor shrinkage on CT scan was observed after NAC. Of the tumor markers, only the CA 19-9 mean value significantly decreased from $183.5 \pm 329.0$ to $93.2 \pm 198.1 \mathrm{IU} / \mathrm{ml}(\mathrm{P}<0.001) .{ }^{18} \mathrm{FDG}-\mathrm{PET} / \mathrm{CT}$ was performed in 34 of 52 patients, before and after NAC. Significant decreases in the SUVmax value from $4.7 \pm 4.2$ to $3.0 \pm 3.2$ were documented after preoperative chemotherapy $(\mathrm{P}=0.003)$. All the tumor specimens showed histopathological evidence of tumor cell injury, although none of the patients exhibited a pathological CR. The NAC treatment effect, as judged by the Evans grading system, was grade I in 41 patients (78.8\%), grade IIa in 8 (15.4\%), and grade IIb in $3(5.8 \%)$. There was no statistically significant difference in the 5-year OS rates of pancreatic head cancer patients in the NAC group compared with the Control group (median survival time (MST): 41.6 months vs. 24.5 months) ( $\mathrm{P}=0.083$; Fig. 2A). OS rate was significantly improved only in node-positive (UICC stage IIB) pancreatic head cancer patients (MST: 29.8 months vs. 19.1 months) ( $\mathrm{P}=0.016$; Fig. 2B). In pancreatic body and tail cancer patients, OS rates of the NAC group were not significantly improved compared with the Control group (MST: 37.2 months vs. 45.2 months) ( $\mathrm{P}=0.772$; Fig. 2C). Subgroup analysis stratified according to postoperative adjuvant chemotherapy demonstrated significant difference only in the 5 -year OS rates of pancreatic head cancer patients. In pancreatic head cancer patients who received postoperative adjuvant chemotherapy, OS rate of the NAC group was significantly improved compared with the Control group ( $\mathrm{P}=0.012$; Fig. 2D).

There were no significant differences in DFS rates of pancreatic head cancer and body and tail cancer patients between the two groups.

Risk factors for early recurrence after surgery. There were 35 (40.7\%) early recurrent PDAC cases within a year after surgery (E group). The remaining 51 cases (L group) consisted of 34 patients without recurrence and 17 patients with recurrence $>1$ year after surgery. In the $\mathrm{E}$ and $\mathrm{L}$ groups, there were no significant differences in gender, age, tumor location, or preand post-operative adjuvant chemotherapy rates. In comparison of tumor size on CT, ${ }^{18} \mathrm{FDG}$ PET SUVmax and tumor marker values as listed in Table III, significantly higher CA19-9 values were observed in the $\mathrm{E}$ group than the $\mathrm{L}$ group $(\mathrm{P}=0.021)$. The cut-off value of CA19-9 values of $30 \mathrm{U} / \mathrm{ml}$ in the NAC group and $88 \mathrm{U} / \mathrm{ml}$ in the Control group were arrived at with ROC analysis. According to the results of the ROC curve analysis, the optimal cut-offs for risk of early recurrence were as follows in the NAC group and the Control group (Fig. 3). When 
Table II. Histopathological characteristics of the NAC and Control groups.

\begin{tabular}{lccr}
\hline Characteristics & NAC group $(\mathrm{n}=52)$ & Control group $(\mathrm{n}=34)$ & P-value \\
\hline Tumor size, mm & $29.8 \pm 15.6$ & $30.4 \pm 10.1$ & 0.862 \\
Serosal invasion, \% & 67.3 & 52.9 & 0.180 \\
Retroperitoneal invasion, \% & 76.9 & 58.8 & 0.074 \\
Lymph node metastasis, \% & 67.3 & 61.8 & 0.598 \\
Neural invasion, \% & 84.6 & 88.2 & 0.636 \\
Plexus invasion, \% & 42.3 & 32.4 & 0.353 \\
R0 rate, \% & 80.8 & 79.4 & 0.901
\end{tabular}

NAC, neoadjuvant chemotherapy.

A

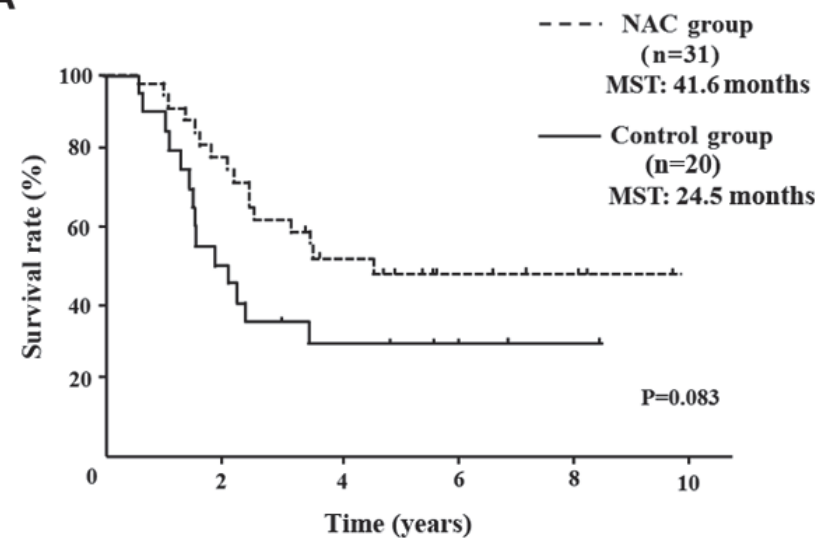

B

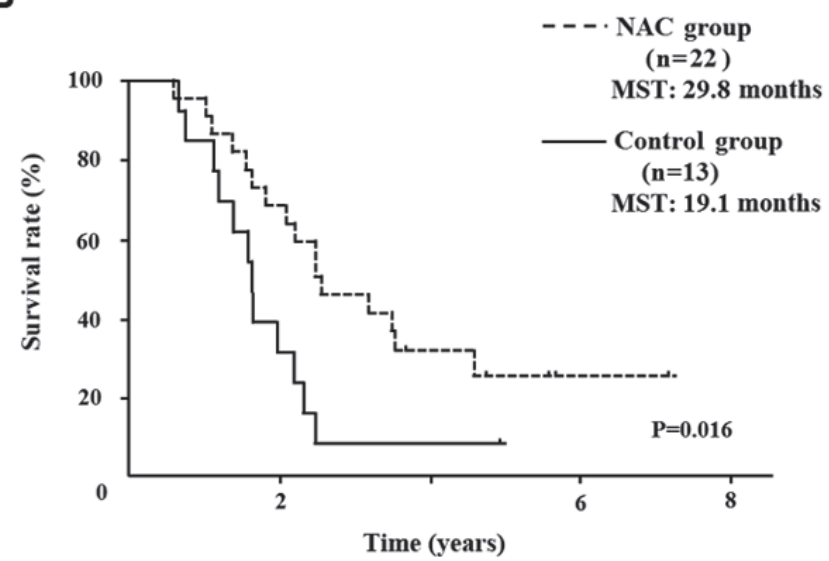

C

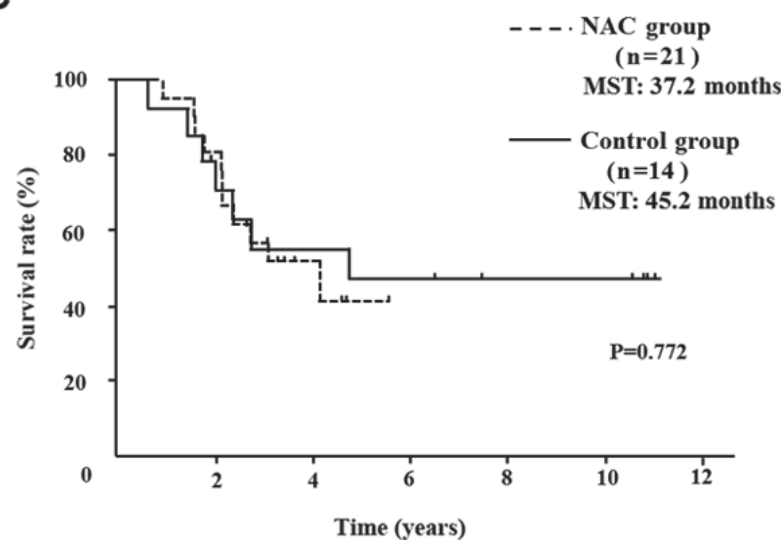

D

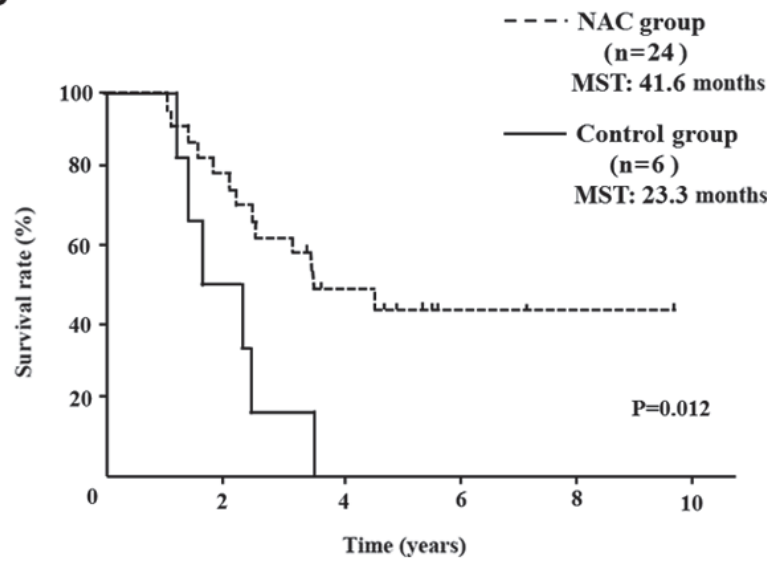

Figure 2. OS curves of patients with PDAC. (A) OS of patients with resectable pancreatic head cancer. The difference was not significant (P=0.124). (B) OS rate of node-positive pancreatic head cancer. OS rate of the NAC group was significantly better than that of the Control group in node positive pancreatic head cancer $(\mathrm{P}=0.016)$. (C) OS rate of pancreatic body and tail cancer. OS rate was not improved with preoperative chemotherapy in patients with pancreatic body and tail cancer. (D) In pancreatic head cancer patients who received postoperative adjuvant chemotherapy, the OS rate of the NAC group was significantly improved compared with the Control group $(\mathrm{P}=0.012)$. PDAC, pancreatic ductal adenocarcinoma; OS, overall survival; NAC, neoadjuvant chemotherapy; MST, median survival time.

CA19-9 in the NAC group was $30.0 \mathrm{U} / \mathrm{ml}$, it had a sensitivity of $47.8 \%$, specificity of $44.8 \%$, positive predictive value (PPV) of $45.8 \%$, and negative predictive value (NPV) of $57.1 \%$, and the area under the ROC curve (AUC) was 0.63 [95\% confidence interval (CI): 0.48-0.79]. When CA19-9 in the Control group was $88 \mathrm{OU} / \mathrm{ml}$, it had a sensitivity of $41.7 \%$, specificity of $54.5 \%$, PPV of $29.4 \%$, and NPV of $58.8 \%$, and the AUC was 0.51 [95\% CI: 0.29-0.74]. In pathological comparison, significantly larger tumor size, higher rates of lymph node metastasis, nerve, and plexus invasion rates were observed in the E group (Table IV). Differences in tumor size were also significant by multivariate analysis. In comparing recurrence sites (Table V), the frequency of liver metastasis and peritoneal dissemination was significantly higher in the E group than in the L group. Of course OS rate of the E group was significantly poor compared with the L group (MST: 55.6 months vs. 20.9 months). However surprisingly, the prognosis of the E group was similar to NAC dropped out patients (MST: 20.0 months). 
Table III. Clinical factors of the E and L groups.

\begin{tabular}{lccr}
\hline Clinical factors & E group $(\mathrm{n}=35)$ & L group $(\mathrm{n}=51)$ & P-value \\
\hline Tumor size on CT, mm & $23.1 \pm 6.3$ & $22.7 \pm 8.1$ & 0.814 \\
${ }^{18}$ FDG-PET SUVmax & $4.8 \pm 3.2$ & $4.0 \pm 3.5$ & 0.403 \\
CEA, ng/ml & $4.0 \pm 5.0$ & $3.4 \pm 3.1$ & 0.468 \\
CA19-9, U/ml & $180.5 \pm 302.2$ & $75.1 \pm 90.0$ & 0.021 \\
DUPAN-2, U/ml & $456.5 \pm 1,833.8$ & $841.1 \pm 4,343.6$ & 0.623
\end{tabular}

${ }^{18}$ FDG-PET SUVmax, 18-fluorodeoxyglucose-positron emission tomography maximum standardized uptake value; CT, computed tomography; CA19-9, carbohydrate antigen 19-9; CEA, carcinoembryonic antigen; DUPAN-2, sialyl-lcat-N-tetraose.

A

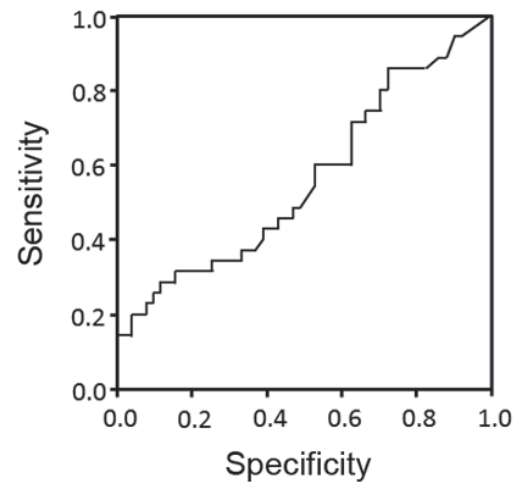

B

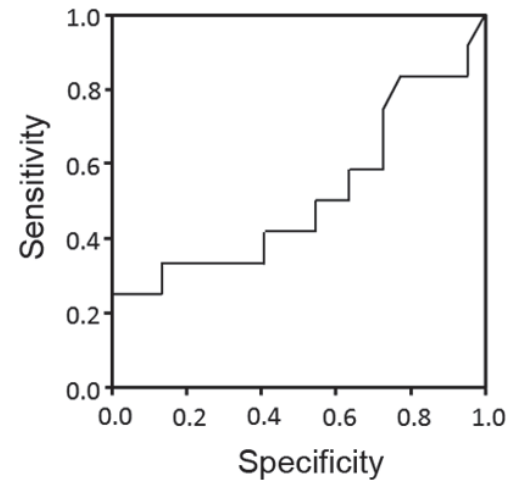

Figure 3. Receiver Operator Characteristic curves for serum carbohydrate antigen 19-9 levels in the (A) NAC group and the (B) Control group. The areas under the curves were 0.63 for the NAC group and 0.51 for the Control group, respectively.

\section{Discussion}

Surgical resection is the only way to cure PDAC. However, the majority of pancreatic cancer resections are reported to be R1 (28), and even after undergoing curative resection, patients with pancreatic cancer face a $50-80 \%$ local recurrence rate and a $25-50 \%$ chance of developing distant metastases (27). We have developed a surgical procedure for PDAC with emphasis on anatomy and embryology $(9,10,26,29)$. Whereby the long-term prognosis of PDAC was improved to some extent $(9,10)$, it is not yet satisfactory even for resectable cases, because of distant metastasis. For this reason, postoperative adjuvant chemotherapy with S-1 (14) was introduced and hepatic arterial infusion chemotherapy with GEM for early postoperative liver metastasis was developed (30-32). Although, the adaptation of NAC to resectable PDAC is still controversial, we made a policy to introduce preoperative chemotherapy even for resectable PDAC to improve treatment outcomes $(23,24)$.

This study retrospectively analyzed patients who underwent resection for resectable PDAC at a single center. Between the NAC group and the Control group, there were no significant differences in patients' clinicopathological characteristics including perioperative factors. We have confirmed all cases are adenocarcinomas, but many cases had plural pathological subtype. Therefore, we did not consider about pathological subtypes of the patients. In the NAC group, significant reduction of CA19-9 value and FDG-PET
SUVmax were observed after preoperative chemotherapy. The effect of NAC according to RECIST guidelines was $\mathrm{SD}$ in $86.5 \%$ cases and the pathological effect judged with Evans grade was I in $78.8 \%$ cases. In the survival analysis of this study, only the patients with node-positive pancreatic head cancer receiving NAC had significantly longer survival time than those in the Control group. Subgroup analysis in postoperative adjuvant chemotherapy group showed that pancreatic head cancer of the NAC group had significantly longer survival time than the Control group. In the analysis of early recurrent cases, there was no correlation with pre- or postoperative chemotherapy; however, a significantly higher CA19-9 value was observed in the E group compared with the $\mathrm{L}$ group $(\mathrm{P}=0.021)$. Moreover, cut-off values of CA19-9 were calculated to be $30 \mathrm{U} / \mathrm{ml}$ in the NAC group and $88 \mathrm{U} / \mathrm{ml}$ in the Control group, respectively, with ROC analysis. In pathological comparison, a significantly higher rate of lymph node metastasis, nerve, and plexus invasion rates were observed in the E group. However, it is difficult to accurately grasp lymph node metastasis and plexus infiltration from preoperative image findings. Even in resectable PDAC cases, if elevated CA19-9 value is not normalized after preoperative chemotherapy, extension of the preoperative chemotherapy period should be considered as for borderline resectable or unresectable PDAC cases $(33,34)$.

The most important purpose of NAC is prevention of metastasis form the primary site and treatment of occult metastasis. However, it has been recently described that 
Table IV. Histopathological features of the E and L groups.

\begin{tabular}{lcccc}
\hline Feature & E group $(\mathrm{n}=35)$ & L group (n=51) & P-value (univariate) & P-value (multivariate) \\
\hline Tumor size, mm & $35.3 \pm 15.7$ & $26.5 \pm 10.8$ & 0.003 & 0.037 \\
Serosal invasion, \% & 65.7 & 58.8 & 0.519 & 0.490 \\
Retroperitoneal invasion, \% & 74.3 & 66.7 & 0.450 & 0.443 \\
Lymph node metastasis, \% & 82.9 & 53.0 & 0.004 & 0.132 \\
Node positive number & 4.2 & 1.7 & 0.005 & 0.980 \\
Neural invasion, \% & 97.1 & 78.4 & 0.014 & 0.159 \\
Plexus invasion, \% & 51.4 & 29.4 & 0.039 & 0.626 \\
R0 rate, \% & 71.4 & 86.3 & 0.089 & 0.714 \\
\hline
\end{tabular}

Table V. Recurrent sites of E and L groups.

\begin{tabular}{lccc}
\hline Site & $\begin{array}{c}\text { E group } \\
(\mathrm{n}=35), \mathrm{n}(\%)\end{array}$ & $\begin{array}{c}\text { L group } \\
(\mathrm{n}=51), \mathrm{n}(\%)\end{array}$ & P-value \\
\hline Liver & $19(54.3)$ & $5(9.8)$ & $<0.0001$ \\
Peritoneum & $11(31.4)$ & $4(7.8)$ & 0.005 \\
Local & $7(22.9)$ & $5(9.8)$ & 0.097 \\
Lung & $3(11.4)$ & $4(7.8)$ & 0.574 \\
Lymph node & $2(5.7)$ & $4(7.8)$ & 0.703 \\
\hline
\end{tabular}

cancer stem cells (CSCs) and cancer associated fibroblasts (CAFs) play an important role in tumor invasion, metastasis, and chemoradioresistance in pancreatic cancer (35). These resistant cells often change the expression of several proteins while acquiring resistance to the therapies. One of the common changes is epithelial mesenchymal transition (EMT). EMT is a biological process that allows epithelial cells to undergo multiple changes, enabling them to assume a mesenchymal cell phenotype, and is positively associated with the malignancy of cancer cells, and their invasiveness, motility, and resistance to apoptosis (36). It has recently been reported that anti-cancer treatments can also induce EMT in cancer cells (37-39). We reported that residual pancreatic cancer tissues resected after preoperative chemotherapy are rich in chemoresistant cancer stem-like cells (40). If this theory is correct, the effectiveness of preoperative therapy for occult metastatic lesions is moot. It will be necessary to select agents with EMT inhibitory effect for the primary lesion during preoperative treatment. It is well known that low-dose paclitaxel (41-44), metformin (45-47), angiotensin receptor blocker $(48,49)$, statins $(50,51)$, and histone deacetylase inhibitors $(52,53)$ have all been indicated as agents that can inhibit the EMT of tumor cells or activation of stromal cells. Many papers have focused on the inhibition of tumor EMT and CAFs activation with paclitaxel $(44,54,55)$. These data corroborate that tumor shrinkage and a decrease in stroma was observed in tumors treated with GnP therapy (56-58). Hence, GnP therapy comprising paclitaxel seems to be most suitable for preoperative chemotherapy theoretically.

Several authors reported that predictors of poor prognosis after surgery for PDAC include early recurrence, elevated serum CA19-9, lymph node metastasis, positive surgical margin $(59,60)$. Serum risk factors for early recurrence have been reported to be elevated Span-1 and CA19-9 $(61,62)$. Kurahara et al (63) reported that serum CA19-9 >85 U/ml was independent risk factor for recurrence within 6 months after upfront surgery. This result is almost the same CA19-9 value (85 U/ml) of the Control group as this study. This study demonstrated that high serum CA19-9, larger tumor size, lymph node metastasis and plexus invasion would be the risk factors for early recurrence after surgery. However, CA19-9 recognition is affected by the patient's Lewis phenotype (64), and preoperative diagnosis of the extent of tumor spread, lymph node metastasis and plexus invasion with CT image is not accurate enough. Even in this study, lymph node metastasis was found to be more than $60 \%$, nerve infiltration was found to be more than $80 \%$, and tumor spread was also larger than preoperative diagnosis in many cases. Therefore, preoperative chemotherapy is considered necessary even for resectable cases, and PDAC with the preoperatively diagnosable risk factors for early recurrence requires preoperative chemotherapy as does borderline resectable PDAC.

In this study period, five patients could not undergo surgery because of tumor progression during preoperative chemotherapy or complication of biliary drainage. In four tumor progression cases, chemotherapy could be continued by changing to another treatment regimen. If upfront surgery was performed in these cases, there is a high possibility that the prognosis was still poor. Preoperative chemotherapy for PDAC may be valuable in the selection of patients without chemoresistant metastases or aggressive local progression. Since this study is a retrospective cohort and included three regimens, prospective randomize study with a fixed regimen is necessary in the future.

In conclusion, NAC with GEM prolonged the survival period of node-positive pancreatic head cancer patients, and SUV max and serum CA19-9 values are useful for judgment of treatment effect. However, high serum CA19-9 value, larger tumor size, lymph node metastasis, and plexus invasion are risk factors for early tumor recurrence after surgery. Especially, in the NAC group normalization of CA19-9 after preoperative chemotherapy will be required. Therefore, preoperative therapy same for borderline resectable cases should be considered even for resectable PDAC cases with early recurrence risk factors. Moreover, postoperative adjuvant chemotherapy is important especially for pancreatic head cancer. 


\section{Acknowledgements}

Not applicable.

\section{Funding}

No funding was received.

\section{Availability of data and materials}

The datasets used and/or analyzed during the present study are available from the corresponding author on reasonable request.

\section{Authors' contributions}

HT and TO designed the present study. HN and SF performed the statistical and pharmacokinetic analyses. MO, TY, SN, JK, IM, YO, KO and KN conducted chemotherapy on patients. TM, HTak and IN contributed to the clinical trial operation. All the authors approved the final version of the manuscript.

\section{Ethics approval and consent to participate}

Written informed consent was obtained from each patient prior to treatment, and the present study was approved by the Ethics Committee of Kanazawa University Hospital (review no. 2799-1).

\section{Patient consent for publication}

Written informed consent was obtained from each patient for the publication of the present study.

\section{Competing interests}

The authors declare that they have no competing interests.

\section{References}

1. Gillen S, Schuster T, Meyer Zum Büschenfelde C, Friess H and Kleeff J: Preoperative/neoadjuvant therapy in pancreatic cancer: A systematic review and meta-analysis of response and resection percentages. PLoS Med 7: e1000267, 2010.

2. Ishii H, Furuse J, Boku N, Okusaka T, Ikeda M, Ohkawa S, Fukutomi A, Hamamoto Y, Nakamura K, Fukuda H, et al: Phase II study of gemcitabine chemotherapy alone for locally advanced pancreatic carcinoma: JCOG0506. Jpn J Clin Oncol 40 573-579, 2010

3. Von Hoff DD, Ramanathan RK, Borad MJ, Laheru DA, Smith LS, Wood TE, Korn RL,Desai N, Trieu V,Iglesias JL, et al: Gemcitabine plus nab-paclitaxel is an active regimen in patients with advanced pancreatic cancer: A phase I/II trial. J Clin Oncol 29: 4548-5454, 2011.

4. Conroy T, Desseigne F, Ychou M, Bouché O, Guimbaud R, Bécouarn Y, Adenis A, Raoul JL, Gourgou-Bourgade S, de la Fouchardière $\mathrm{C}$, et al: FOLFIRINOX versus gemcitabine for metastatic pancreatic cancer. N Engl J Med 364: 1817-1825, 2011.

5. Wagner M, Redaelli C, Lietz M, Seiler CA, Friess H and Búcher MW: Curative resection is the single most important factor determining outcome in patients with pancreatic adenocarcinoma. Br J sSurg 91: 586-594, 2004.

6 . Verbeke CS and Menon KV: Redefining resection margin status in pancreatic cancer. HPB (Oxford) 11: 282-289, 2009.

7. Nagakawa T, Kurachi M, Konishi K and Miyazaki I: Translateral retroperitoneal approach in radical surgery for pancreatic carcinoma. Jpn J Surg 12: 229-33, 1982.
8. Nagakawa T, Nagamori M, Futakami F, Tsukioka Y, Kayahara M, Ohta T, Ueno K and Miyazaki I: Result of extensive surgery for pancreatic carcinoma. Cancer 77: 640-645, 1996.

9. Kitagawa H, Tajima H, Nakagawara H, Makino I, Miyashita T, Shoji M, Nakanuma S, Hayashi N, Takamura H, Ohta T and Ohtake $\mathrm{H}$ : En bloc vascular resection for the treatment of borderline resectable pancreatic head carcinoma. Mol Clin Oncol 2: 369-374, 2014

10. Kitagawa H, Tajima H, Nakagawara H, Makino I, Miyashita $T$, Terakawa H, Nakanuma S, Hayashi $\mathrm{H}$, Takamura $\mathrm{H}$ and Ohta T: A modification of radical antegrade modular pancreato-splenectomy for adenocarcinoma of the left pancreas: Significance of en bloc resection including the anterior renal fascia. World J Surg 38: 2448-2454, 2014

11. Nimura Y, Nagino M, Takao S, Takada T, Miyazaki K, Kawarada Y Miyagawa S, Yamaguchi A, Ishiyama S, Takeda Y, et al: Standard versus extended lymphadenectomy in radical pancreato-duodenectomy for ductal adenocarcinoma of the head of the pancreas: Long-term results of a Japanese multicenter randomized controlled trial. J Hepatobiliary Pancreat Sci 19: 230-2341, 2012.

12. Evans DB, Abbruzzese JL and Willett CG: Cancer of the pancreas. In: DeVita VT, Hellman S and Rosenberg SA (eds.): Cancer: Priciples and Practice of Oncology. 6th edition. Philadelphia, Lippincott, Williams and Wilkins 1: pp1126-1161, 1997.

13. Oettle H, Post S, Neuhaus P, Gellert K, Langrehr J, Ridwelski K, Schramm H, Fahlke J, Zuelke C, Burkart C, et al: Adjuvant chemotherapy with gemcitabine vs observation in patients undergoing curative-intent resection of pancreatic cancer: A randomized controlled trial. JAMA 297: 267-277, 2007.

14. UesakaK,BokuN,FukutomiA,OkamuraY,KonishiM,MatsumotoI, Kaneoka Y, Shimizu Y, Nakamori S, Sakamoto H, et al: Adjuvant chemotherapy of S-1 versus gemcitabine for resected pancreatic cancer: A phase 3, open-label, randomised, non-inferiority trial (JASPAC 01). Lancet 388: 248-257, 2016.

15. Klinlenbijl JH, Jeekel J, Sahmoud T, van Pel R, Couvreur ML, Veenhof CH, Arnaud JP, Gonzalez DG, de Wit LT, Hennipman A, et al: Adjuvant radiotherapy and 5-fluorouracil after curative resection of cancer of the pancreas and periampullary region: Phase III trial of the EORTC gastrointestinal tract cancer cooperative group. Ann Surg 230: 776-782, 1999.

16. Spitz FR, Abbruzzese JL, Lee JE, Pisters PW, Lowy AM, Fenoglio CJ, Cleary KR, Janjan NA, Goswitz MS, Rich TA, et al: Preoperative and postoperative chemoradiation strategies in patients treated with pancreaticoduodenectomy for adenocarcinoma of the pancreas. J Clin Oncol 15: 928-937, 1997.

17. Yeo CJ, Abrams RA, Grochow LB, Sohn TA, Ord SE, Hruban RH, Zahurak ML, Dooley WC, Coleman J, Sauter PK, et al: Pancreaticoduodenectomy for pancreatic adenocarcinoma: Postoperative adjuvant chemoradiation improves survival. A prospective, single-institution experience. Ann Surg 225: 621-633, 1997.

18. Lee JL, Kim SC, Kim JH, Lee SS, Kim TW, Park DH, Seo DW, Lee SK, Kim MH, Kim JH, et al: Prospective efficacy and safety study of neoadjuvant gemcitabine with capecitabine combination chemotherapy for borderline-resectable or unresectable locally advanced pancreatic adenocarcinoma. Surgery 152: 851-862, 2012

19. Itchins M, Arena J, Nahm CB, Rabindran J, Kim S, Gibbs E, Bergamin S, Chua TC, Gill AJ, Maher R, et al: Retrospective cohort analysis of neoadjuvant treatment and survival in resectable and borderline resectable pancreatic ductal adenocarcinoma in a high volume referral center. Eur J Surg Oncol 43: 1711-1717, 2017.

20. Katz MH, Shi Q, Ahmad SA, Herman JM, Marsh Rde W, Collisson E, Schwartz L, Frankel W, Martin R, Conway W, et al: Preoperative modified FOLFIRINOX treatment followed by capecitabine-based chemoradiation for borderline resectable pancreatic cancer: Alliance for clinical trials in oncology Trial A021101. JAMA Surg 151: e161137, 2016.

21. Shubert CR, Bergquist JR, Groeschl RT, Habermann EB, Wilson PM, Truty MJ, Smoot RL, Kendrick ML, Nagorney DM and Farnell MB: Overall survival is increased among stage III pancreatic adenocarcinoma patients receiving neoadjuvant chemotherapy compared to surgery first and adjuvant chemotherapy: An intention to treat analysis of the National Cancer Database. Surgery 160: 1080-1096, 2016.

22. Nagakawa Y, Hosokawa Y, Nakayama H, Sahara Y, Takishita C, Nakajima T, Hijikata Y, Kasuya K, Katsumata K, Tokuuye K and Tsuchida A: A phase II trial of neoadjuvant chemoradiotherapy with intensity-modulated radiotherapy combined with gemcitabine and S-1 for borderline-resectable pancreatic cancer with arterial involvement. Cancer Chemother Pharmacol 79: 951-957, 2017. 
23. Tajima H, Ohta T, Kitagawa H, Okamoto K, Sakai S, Makino I, Kinoshita J, Furukawa H, Nakamura K, Hayashi H, et al: Pilot study of neoadjuvant chemotherapy with gemcitabine and oral S-1 for resectable pancreatic cancer. Exp Ther Med 3: 787-792, 2012.

24. Tajima H, Kitagawa H, Tsukada T, Nakanuma S, Okamoto K, Sakai S, Makino I, Furukawa H, Nakamura K, Hayashi H, et al: A phase I study of neoadjuvant chemotherapy with gemcitabine plus oral S-1 for resectable pancreatic cancer. Mol Clin Oncol 1: 768-772, 2013.

25. Therasse P, Arbuck SG, Eisenhauer EA, Wanders J, Kaplan RS, Rubinstein L, Verweij J, Van Glabbeke M, van Oosterom AT, Christian MC and Gwyther SG: New guidelines to evaluate the response to treatment in solid tumors. J Natl Cancer Inst 92 205-216, 2000.

26. Makino I, Kitagawa H, Ohta T, Nakagawara H, Tajima H, Ohnishi I, Takamura H, Tani T and Kayahara M: Nerve plexus invasion in pancreatic cancer. Spread patterns on histopathologic and embryological analysis. Pancreas 37: 358-365, 2008.

27. Evans DB, Rich TA, Byrd DR, Cleary KR, Connelly JH, Levin B, Charnsangavej C, Fenoglio CJ and Ames FC: Preoperative chemoradiation and pancreaticoduodenectomy for adenocarcinoma of the pancreas. Arch Surg 127: 1335-1339, 1992.

28. Esposito I, Kleeff J, Bergmann F, Reiser C, Herpel E, Friess H, Schirmacher P and Büchler MW: Most pancreatic cance resections are R1 resections. Ann Surg Oncol 15: 1651-1660, 2008

29. Terakawa H, Kitagawa H, Makino I, Hayashi H, Oyama K, Nakagawara H, Miyashita T, Tajima H, Takamura H, Fushida S, et al: Location of the meso-pancreatoduodenum as a regional lymphatic basin for pancreatic head carcinoma. Oncol Lett 14: 397-403, 2017.

30. Tajima H, Ohta T, Kitagawa H, Sakai S, Makino I, Hayashi H, Nakagawara H, Onishi I, Takamura H, Ninomiya I, et al: Hepatic arterial infusion chemotherapy for post-operative liver metastases from pancreatic cancer in a patient with leukocytopenia: A case report. Exp Ther Med 1: 987-990, 2010.

31. Tajima H, Ohta T, Kitagawa H, Sakai S, Makino I, Hayashi H, Oyama K, Nakagawara H, Fujita H, Onishi I, et al: Pilot study of hepatic arterial infusion chemotherapy with gemcitabine and 5-fluorouracil for patients with postoperative liver metastases from pancreatic cancer. Exp Ther Med 2: 265-269, 2011.

32. Tajima H, Kitagawa H, Tsukada T, Okamoto K, Nakanuma SI, Sakai S, Makino I, Furukawa $\mathrm{H}$, Hayashi $\mathrm{H}$, Oyama $\mathrm{Ku}$, et al: Hepatic arterial infusion chemotherapy with gemcitabine and 5 -fluorouracil or oral S-1 improves the prognosis of patients with postoperative liver metastases from pancreatic cancer. Mol Clinic Oncol 1: 869-874, 2013.

33. Satoi S, Yamaue H, Kato K, Takahashi S, Hirono S, Takeda S, Eguchi H, Sho M, Wada K, Shinchi H, et al: Role of adjuvant surgery for patients with initially unresectable pancreatic cancer with a long-term favorable response to non-surgical anti-cancer treatments: Results of a project study for pancreatic surgery by the Japanese society of hepato-biliary-pancreatic surgery. J Hepatobiliary Pancreat Sci 20: 590-600, 2013.

34. Eguchi H, Yamada D, Iwagami Y, Gotoh K, Kawamoto K, Wada H, Asaoka T, Noda T, Takeda Y, Tanemura M, et al: Prolonged neoadjuvant therapy for locally advanced pancreatic cancer. Dig Surg 35: 70-76, 2018.

35. Valle S, Martin-Hijano L, Alcalá S, Alonso-Nocelo $M$ and Sainz B Jr: The ever-evolving concept of the cancer stem cell in pancreatic cancer. Cancers (Basel) 10: pii: E33, 2018.

36. Tsubouchi K, Minami K, Hayashi N, Yokoyama Y, Mori S, Yamamoto $\mathrm{H}$ and Koizumi M: The CD44 standard isoform contributes to radioresistance of pancreatic cancer cells. J Radiat Res 58: 816-826, 2017.

37. Yang AD, Fan F, Camp ER, van Buren G, Liu W, Somcio R, Gray MJ, Cheng H, Hoff PM and Ellis LM: Chronic oxaliplatin resistance induces epithelial-mesenchymal transition in colorectal cancer cell lines. Clin Cancer Res 12: 4147-4153, 2006.

38. Tsukamoto H, Shibata K, Kajiyama H, Terauchi M, Nawa A and Kikkawa F: Irradiation-induced epithelial-mesencymal transition (EMT) related to invasive potential in endometrial carcinoma cells. Gynecol Oncol 107: 500-504, 2007.

39. Tajima H, Ohta T, Makino I, Hayashi H, Nakagawara $H$, Onishi I, Takamura H, Ninomiya I, Kitagawa H, Fushida S, et al: Expression of epithelial-mesenchymal transition markers in locally recurrent hepatocellular carcinoma after radiofrequency ablation. Exp Therap Med 1: 347-350, 2010.
40. Tajima H, Ohta T, Kitagawa H, Okamoto K, Sakai S, Kinoshita J, Makino I, Furukawa H, Hayashi H, Nakamura K, et al: Neoadjuvant chemotherapy with gemcitabine for pancreatic cancer increases in situ expression of the apoptosis marker M30 and stem cell marker CD44. Oncol Lett 3: 1186-1190, 2012

41. Zhang D, Sun L, Xian W, Liu F, Ling G, Xiao L, Liu Y, Peng Y, Haruna Y and Kanwar YS: Low-dose paclitaxel ameliorates renal fibrosis in rat UUO model by inhibition of TGF-beta/Smad activity. Lab Invest 90: 436-447, 2010

42. Zhou J, Zhong DW, Wang QW, Miao XY and Xu XD: Paclitaxel ameliorates fibrosis in hepatic stellate cells via inhibition of TGF-beta/Smad activity. World J Gastroenterol 16: 3330-3334, 2010.

43. Choi HS, Savard CE, Choi JW, Kuver R and Lee SP: Paclitaxel interrupts TGF-beta1 signaling between gallbladder epithelial cells and myofibroblasts. J Surg Res 141: 183-191, 2007.

44. Hirose A, Tajima H, Ohta T, Tsukada T, Okamoto K, Nakanuma S, Sakai S, Kinoshita J, Makino I, Furukawa H, et al: Low-dose paclitaxel inhibits the induction of epidermal-mesenchymal transition in the human cholangiocarcinoma CCKS-1 cell line. Oncol Lett 6: 915-920, 2013.

45. Cufí S, Vazquez-Martin A, Oliveras-Ferraros C, MartinCastillo B, Joven J and Menendez JA: Metformin against TGF $\beta$-induced epithelial-to- mesenchymal transition (EMT): From cancer stem cells to aging-associated fibrosis. Cell Cycle 9: 4461-4468, 2010

46. Vazquez-Martin A, Oliveras-Ferraros C, Cufí S, Del Barco S, Martin-Castillo B and Menendez JA: Metformin regulates breast cancer stem cell ontogeny by transcriptional regulation of the epithelial-mesenchymal transition (EMT) status. Cell Cycle 9: 3807-3814, 2010

47. Del Barco S, Vazquez-Martin A, Cufí S, Oliveras-Ferraros C, Bosch-Barrera J, Joven J, Martin-Castillo B and Menendez JA: Metformin: Multi-faceted protection against cancer. Oncotarget 2: 896-917, 2011.

48. Okamoto K, Tajima H, Ohta T, Nakanuma S, Hayashi H, Nakagawara H, Onishi I, Takamura H, Ninomiya I, Kitagawa H, et al: Angiotensin II induces tumor progression and fibrosis in intrahepatic cholangiocarcinoma through an interaction with hepatic stellate cells. Int J Oncol 37: 1251-1259, 2010.

49. Okazaki M,Fushida S, Harada S, Tsukada T, Kinoshita J, Oyama K, Tajima H, Ninomiya I, Fujimura T and Ohta T: The angiotensin II type 1 receptor blocker candesartan suppresses proliferation and fibrosis in gastric cancer. Cancer Lett 355: 46-53, 2014.

50. Chang TI, Kang HY, Kim KS, Lee SH, Nam BY, Paeng J, Kim S, Park JT, Yoo TH, Kang SW and Han SH: The effect of statin on epithelial-mesenchymal transition in peritoneal mesothelial cells. PLoS One 9: e109628, 2014.

51. Yang $T$, Chen $M$ and Sun $T$ : Simvastatin attenuates TGF- $\beta 1$-induced epithelial-mesenchymal transition in human alveolar epithelial cells. Cell Physiol Biochem 31: 863-874, 2013.

52. Shoji M, Ninomiya I, Makino I, Kinoshita J, Nakamura K, Oyama K, Nakagawara H, Fujita H, Tajima H, Takamura H, et al: Valproic acid, a histone deacetylase inhibitor, enhances radiosensitivity in esophageal squamous cell carcinoma. Int J Oncol 40: 2140-2146, 2012.

53. Watanabe T, Tajima H, Hironori H, Nakagawara H, Ohnishi I, Takamura $\mathrm{H}$, Ninomiya I, Kitagawa $\mathrm{H}$, Fushida S, Tani T, et al: Sodium valproate blocks the transforming growth factor (TGF)- $\beta 1$ autocrine loop and attenuates the TGF- $\beta 1$-induced collagen synthesis in a human hepatic stellate cell line. Int J Mol Med 28: 919-925, 2011.

54. Sun JD, Liu Q, Ahluwalia D, Li W, Meng F, Wang Y, Bhupathi D, Ruprell AS and Hart CP: Efficacy and safety of the hypoxiaactivated prodrug TH-302 in combination with gemcitabine and nab-paclitaxel in human tumor xenograft models of pancreatic cancer. Cancer Biol Ther 16: 438-449, 2015.

55. Zhang D, Yang R, Wang S and Dong Z: Paclitaxel: New uses for an old drug. Drug Des Devel Ther 8: 279-284, 2014

56. Meng H, Wang M, Liu H, Liu X, Situ A, Wu B, Ji Z, Chang CH and Nel AE: Use of a lipid-coated mesoporous silica nanoparticle platform for synergistic gemcitabine and paclitaxel delivery to human pancreatic cancer in mice. ACS Nano 9: 3540-3557, 2015.

57. Oettle H: Progress in the knowledge and treatment of advanced pancreatic cancer: From benchside to bedside. Cancer Treat Rev 40: 1039-1047, 2014.

58. Miyashita T, Tajima H, Makino I, Okazaki M, Yamaguchi T, Ohbatake Y,Nakanuma S, Hayashi H, Takamura H, Ninomiya I, et al: Neoadjuvant chemotherapy with gemcitabine plus nab-paclitaxel reduces the number of cancer-associated fibroblasts through depletion of pancreatic stroma. Anticancer Res 38: 337-343, 2018. 
59. Waraya M, Yamashita K, Katagiri H, Ishii K, Takahashi Y, Furuta K and Watanabe M: Preoperative serum CA19-9 and dissected peripancreatic tissue margin as determiners of long-term survival in pancreatic cancer. Ann Surg Oncol 16: 1231-1240, 2009.

60. Ueda M, Endo I, Nakashima M, Minami Y, Takeda K, Matsuo K, Nagano Y, Tanaka K, Ichikawa Y, Togo S, et al: Prognostic factors after resection of pancreatic cancer. World J Surg 33: 104-110, 2009.

61. Hosokawa Y, Nagakawa Y, Sahara Y, Takishita C, Katsumata K and Tsuchida A: Serum SPan-1 is a significant risk factor for early recurrence of pancreatic cancer after curative resection. Dig Surg 34: 125-132, 2017.
62. Shimizu T, Asakuma M, Tomioka A, Inoue Y, Hirokawa F, Hayashi M and Uchiyama K: Span-1 and CA19-9 as predictors of early recurrence and lymph node metastasis for patients with invasive pancreatic cancer after pancreatectomy. Am Surg 84: 109-113, 2018.

63. Kurahara H, Maemura K, Mataki Y, Sakoda M, Iino S, Kawasaki Y, Arigami T, Mori S, Kijima Y, Ueno S, et al: A Therapeutic strategy for resectable pancreatic cancer based on risk factors of early recurrence. Pancreas 47: 753-758, 2018.

64. Berge AC, Meszoely IM, Ross EA, Watson JC and Hoffman JP: Undetectable preoperative levels of serum CA 19-9 correlate with improved survival for patients with resectable pancreatic adenocarcinoma. Ann Surg Oncol 11: 644-649, 2004. 